Supporting Information

\title{
Near-Infrared Photoluminescence Properties of Endohedral Mono- and Di-Thulium Metallofullerenes
}

Zhiyong Wang, ${ }^{\dagger}$ Noriko Izumi, ${ }^{\dagger}$ Yusuke Nakanishi, ${ }^{\dagger}$ Takeshi Koyama,${ }^{\ddagger}$ Toshiki Sugai ${ }^{\dagger}$ Masayoshi Tange, ${ }^{\S}$ Toshiya Okazaki, ${ }^{\S}$ and Hisanori Shinohara ${ }^{*}$,

${ }^{\dagger}$ Department of Chemistry and Institute for Advanced Research, Nagoya University, Nagoya 464-8602, Japan.

'Department of Applied Physics, Nagoya University, Nagoya 464-8603, Japan.

${ }^{\S}$ Research Center for Advanced Carbon Materials, National Institute of Advanced Industrial Science and Technology (AIST), Tsukuba 305-8565, Japan. 


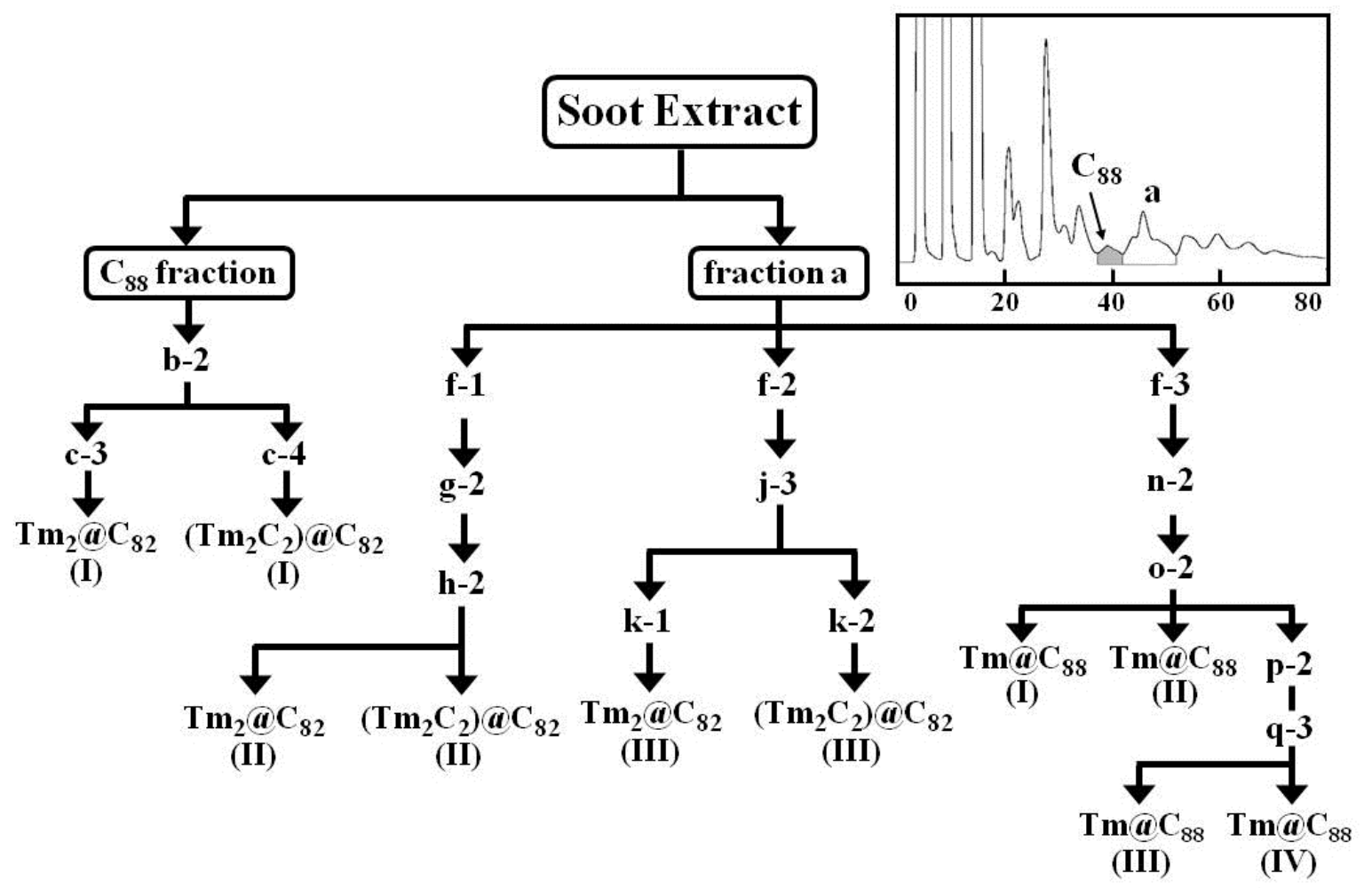

Figure S1. The overall HPLC separation scheme for thulium metallofullerenes. Toluene was used as the eluent. 

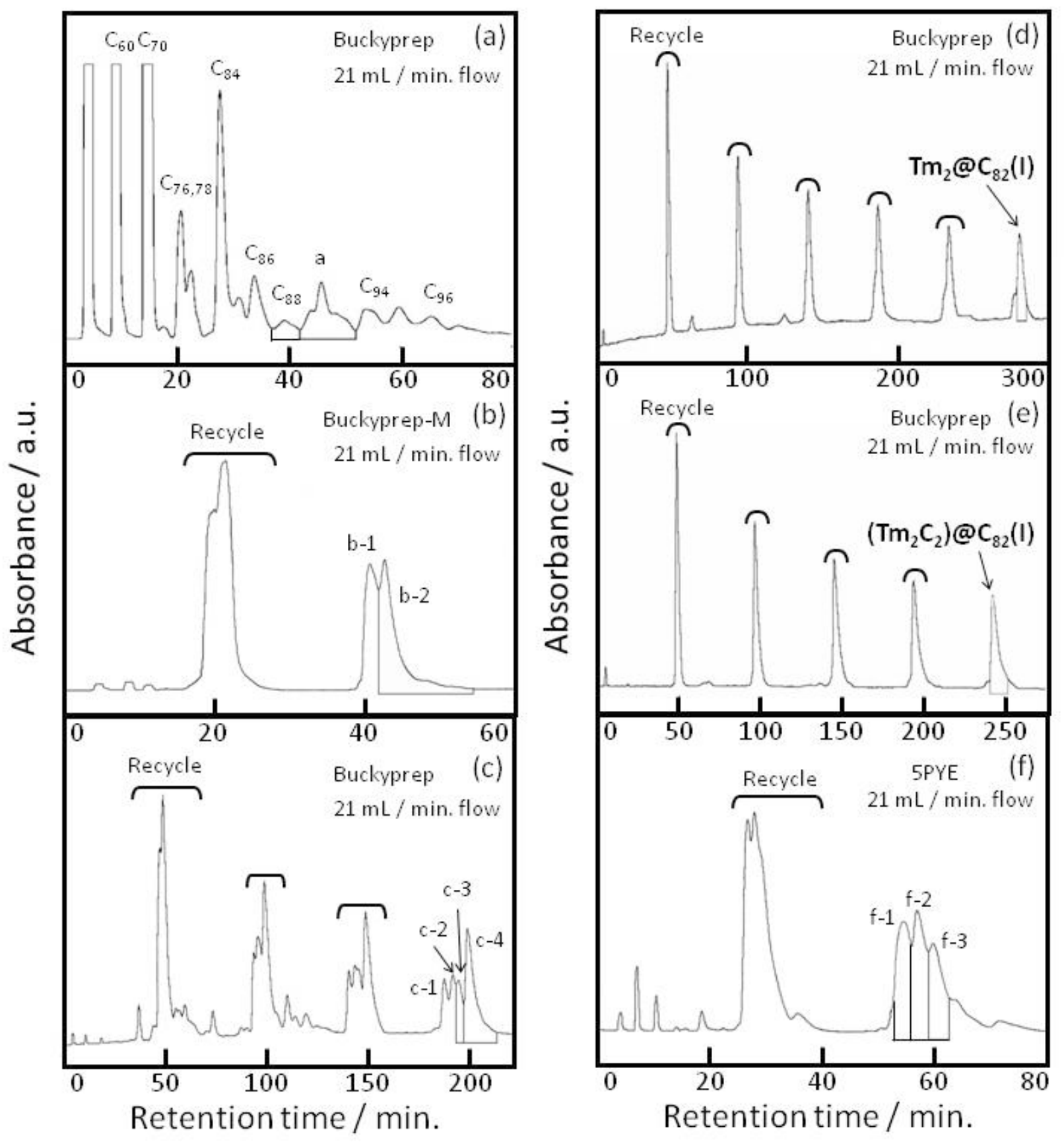

Figure S2. The HPLC separation procedure (1). 

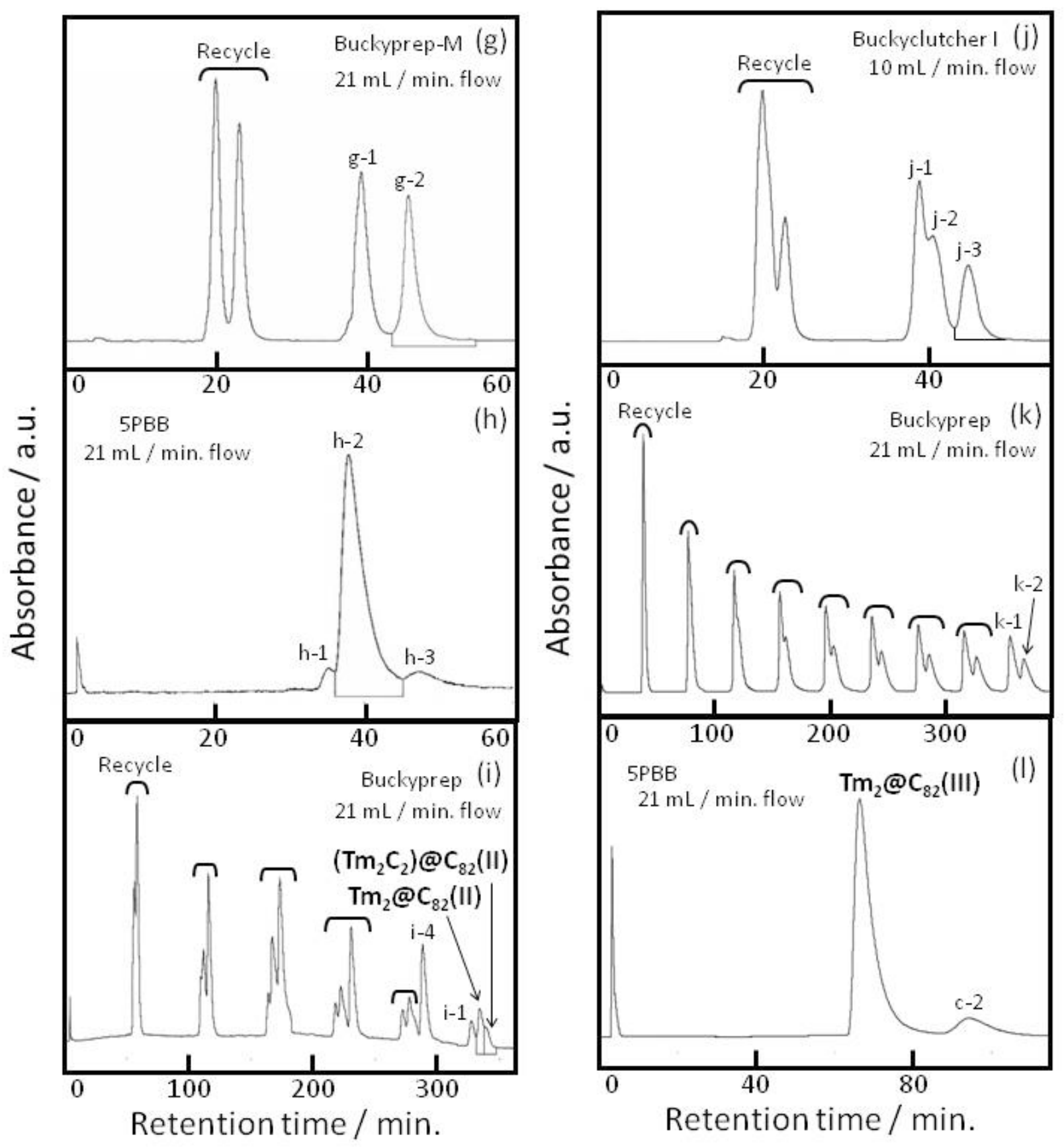

Figure S3. The HPLC separation procedure (2). 

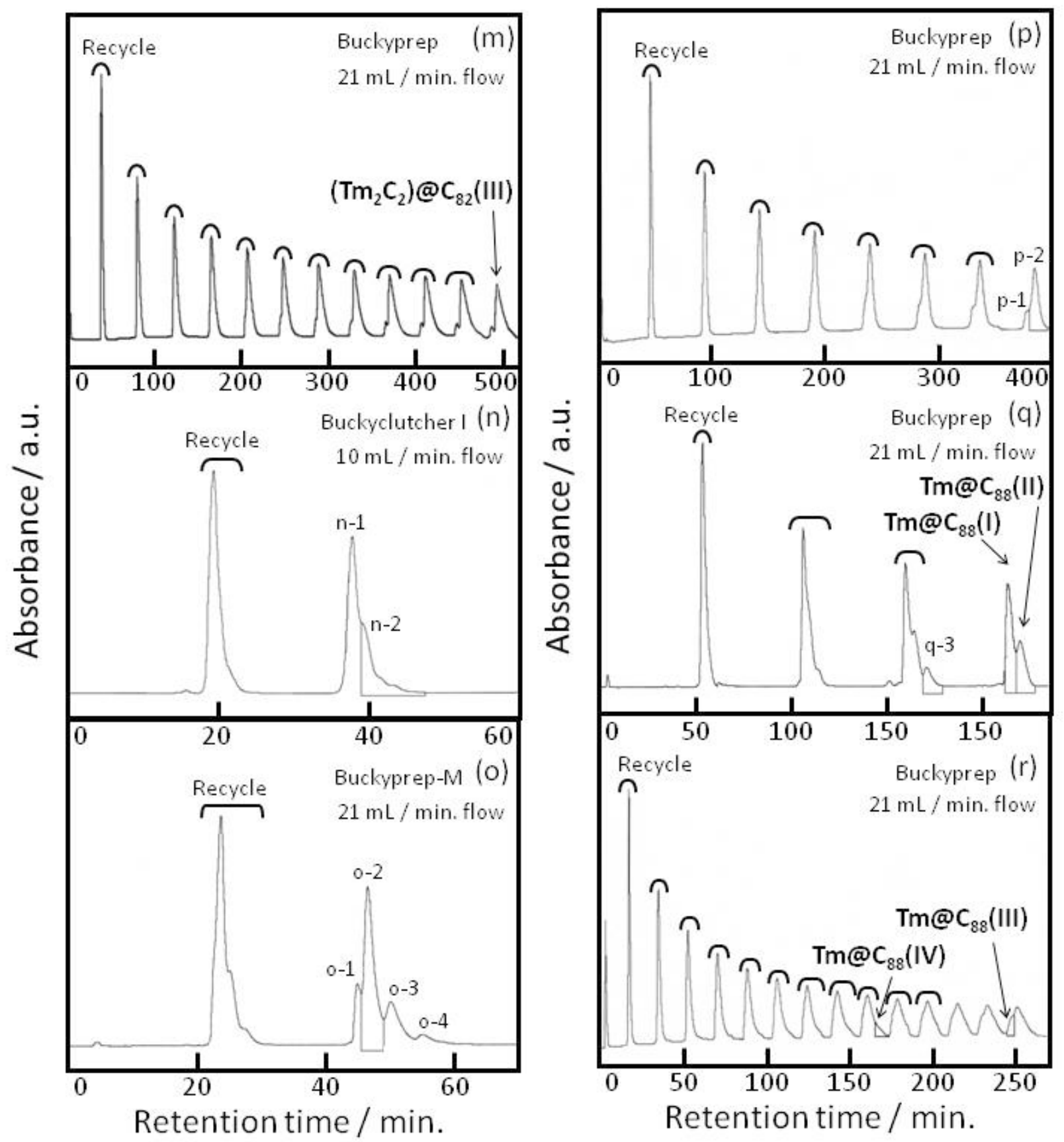

Figure S4. The HPLC separation procedure (3). 

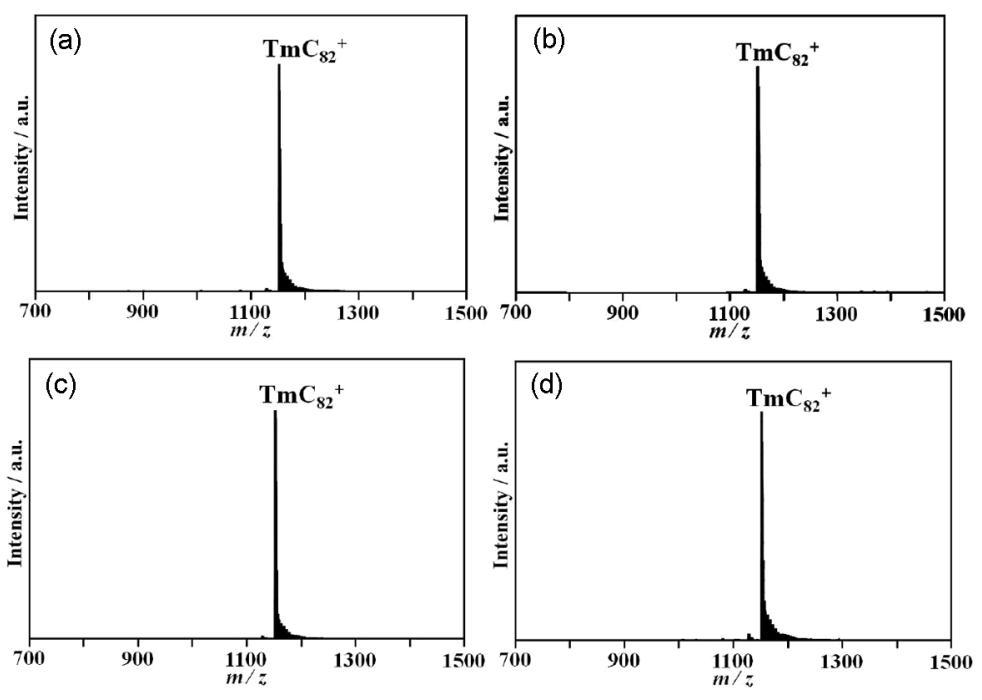

Figure S5. (a, b, c, d) LD-TOF mass spectra of Tm@ $\mathrm{C}_{82}$ (I, II, III, IV).
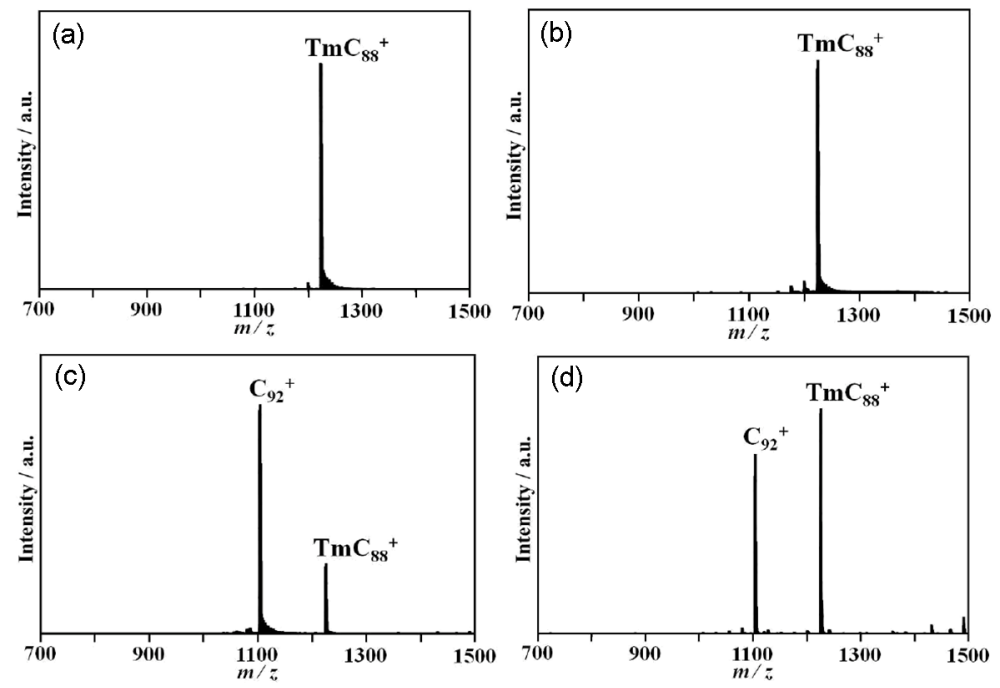

Figure S6. (a, b, c, d) LD-TOF mass spectra of Tm@ $\mathrm{C}_{88}$ (I, II, III, IV). The samples of Tm@ $\mathrm{C}_{88}$ (III, IV) contain empty fullerene $\mathrm{C}_{92}$. 

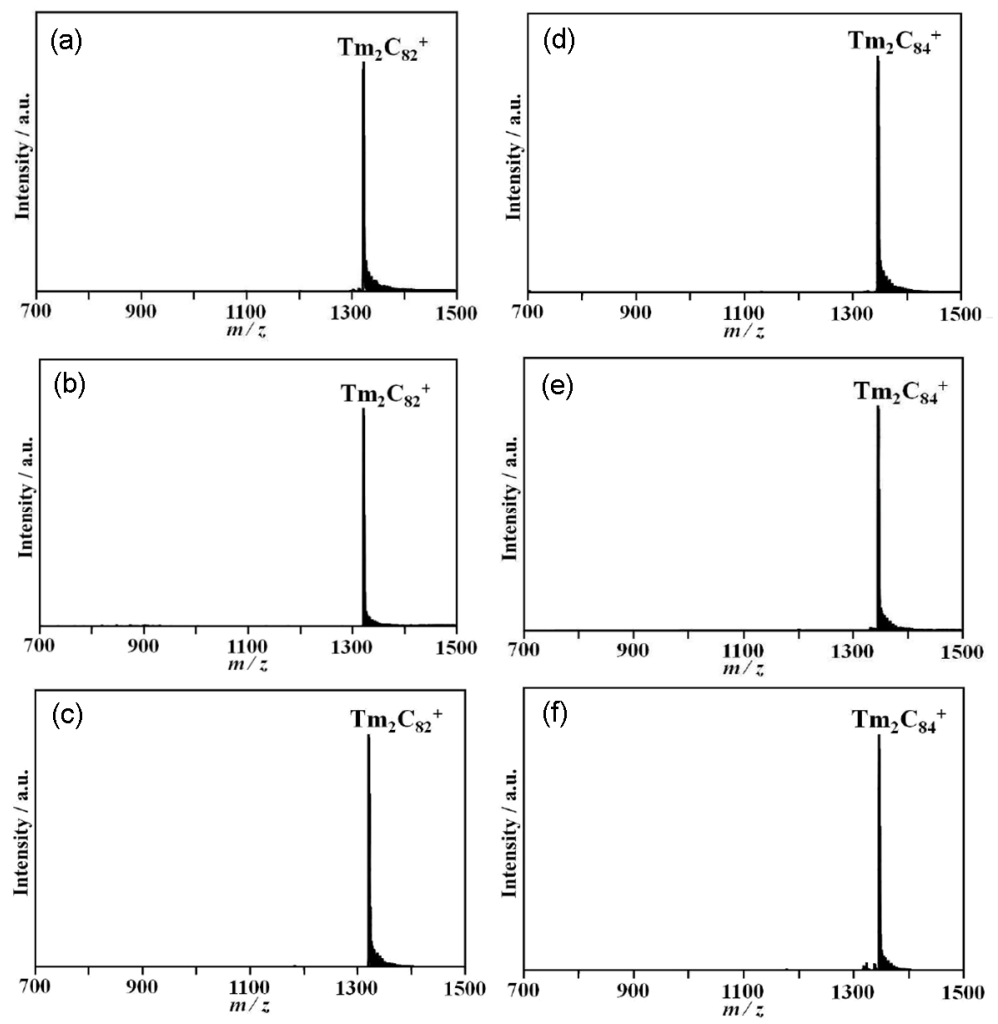

Figure S7. (a, b, c) LD-TOF mass spectra of Tm $2 @ \mathrm{C}_{82}$ (I, II, III). (d, e, f) LD-TOF mass spectra of $\mathrm{Tm}_{2} \mathrm{C}_{2} @ \mathrm{C}_{82}$ (I, II, III). 